\title{
Potential Significance and Clinical Value Explorations of Calmin (CLMN) in Breast Invasive Carcinoma
}

\author{
Yan Wu' \\ Chun-Ping Liu ${ }^{2}$ \\ Cheng Xiang ${ }^{3}$ \\ Kai-Fang Xiang ${ }^{4}$ \\ 'Department of Oncology, The Sixth \\ Hospital of Wuhan, Affiliated Hospital of \\ Jianghan University, Hubei, 430019 , \\ Wuhan, People's Republic of China; \\ ${ }^{2}$ Department of Thyroid and Breast \\ Surgery, The Union Hospital, Tongji \\ Medical College, Huazhong University of \\ Science and Technology, Hubei, Wuhan, \\ People's Republic of China; ${ }^{3}$ Department \\ of Thyroid Surgery, The Second Affiliated \\ Hospital, Zhejiang University School of \\ Medicine, Hangzhou, 310009, Zhejiang, \\ People's Republic of China; ${ }^{4}$ Department \\ of Thyroid and Breast Surgery, The Union \\ Jiangnan Hospital, Huazhong University of \\ Science and Technology, Wuhan, 430200, \\ Hubei, People's Republic of China
}

Correspondence: Kai-Fang Xiang Department of Thyroid and Breast Surgery, The Union Jiangnan Hospital, Huazhong University of Science and Technology, No.I Wenhua Avenue, Jiangxia District, Wuhan, 430200, Hubei, People's Republic of China

Tel +86-27-15827466657

Email xkf050I@I26.com
Objective: Function of calmin (CLMN) was rarely reported in human diseases, especially in tumor. Present study initially assessed the significance of CLMN in breast invasive carcinoma (BRCA).

Methods: Expressions of CLMN containing mRNA and protein in BRCA was firstly assessed, and association of CLMN mRNA expression with clinical phenotypes of BRCA patients was analyzed as well. Prognostic value of CLMN in BRCA was subsequently predicted based on the clinical characteristics of patients. Finally, the potential biological function associated with CLMN involved in BRCA was revealed.

Results: (1) The mRNA expression of CLMN was lower in BRCA compared with that in normal patients $(P<0.001)$. However, result of CLMN total protein expression was opposite $(P<0.05)$. (2) The mRNA expression of CLMN was statistically associated with BRCA patient's age, gender, PR status, ER status, histological type, tumor stage, copy number, and methylation level (all $P<0.05$ ). (3) Compared with low expression group, high expression of CLMN was conducive to the overall survival of BRCA patients $(P=0.0011)$. Detailed, survival difference between CLMN high and low expression groups was observed in patients with stage $1(P=0.0250)$, positive ER status $(P=0.0042)$, negative HER status $(P=0.0433)$, luminal A $(P=0.0065)$, luminal $\mathrm{B}(P=0.0123)$ and positive lymph node status $(P=0.0069)$. Pathway analysis suggested that CLMN mainly participated in cell cycle process $(P<0.05)$ and exerted inhibition effect on the cell cycle involved in BRCA $(P<0.05)$.

Conclusion: CLMN mRNA high expression prolonged the survival time of patients and caused a favorable prognosis. The positive function of CLMN in BRCA required further investigation in future work.

Keywords: CLMN, breast invasive carcinoma, prognosis, pathway, cell cycle

\section{Introduction}

Breast invasive carcinoma (BRCA) showed the highest incidence rate among female cancer patients and posed a great threat to women's health. ${ }^{1}$ The main methods for BRCA treatment included surgery, chemotherapy, radiation therapy, targeted therapy, and endocrine therapy. ${ }^{2}$ However, patients with metastatic or advanced breast cancer were not insensitive to these therapies. Despite that the prognosis of patients has been improved over the past few years, the complex biological behaviors of breast cancer still hamper the progress in clinical treatment. ${ }^{3}$ Therefore, it was of great importance to illuminate the exact molecular mechanisms 
of cancer progression in order to further develop more efficient diagnostic means as well as therapeutic options.

Calmin (CLMN) was identified as one of all-trans retinoic acid (atRA)-responsive genes. ${ }^{4}$ atRA, a vitamin A metabolite, was a regulator of nervous system development. The transcript of CLMN can be detected early in embryonic development and was sensitive to retinoid status. In terms of vitamin A-deficient, CLMN mRNA was dramatically downregulated in the neuroepithelium adjacent to the somites, and the down-regulation can be rescued with the addition of atRA. ${ }^{5}$ CLMN has been proven to involve in the development and/or maintenance of neuronal functions. ${ }^{6}$ At present, very few studies reported the function of CLMN in human diseases, especially in tumor. Therefore, it was necessary to explore the potential function of CLMN in BRCA.

Present study initially revealed the important role of CLMN in breast invasive carcinoma. We first evaluated the expression of CLMN in BRCA containing mRNA and protein levels. The genetic alteration of CLMN in BRAC was then explored. Prognostic impact caused by CLMN expression on patients with BRCA was subsequently assessed. Finally, the possible mechanism associated with CLMN involved in BRCA was disclosed.

\section{Methods}

\section{CLMN mRNA and Protein Expression}

\section{Analyses}

The mRNA expression of CLMN in human cancer cell lines was evaluated through Cancer Cell Line Encyclopedia (CCLE) database (https://portals.broadinsti tute.org/ccle/). The differential expression of CLMN mRNA in normal and breast invasive carcinoma tissues was assessed through UALCAN database (http://ualcan. path.uab.edu/). Subsequently, protein expression overview of CLMN in human cancers, immunohistochemical images in normal and breast cancer tissues, and main location within cancer cell were obtained from Human Protein Atlas (HPA) database. In addition, UALCAN database was also used to perform quantitative analysis on CLMN total protein based on CPTAC samples between normal and BRCA patients.

\section{Association Analysis Between CLMN mRNA Expression and Related Factors}

The association of CLMN mRNA expression with clinical phenotypes of patients was then analyzed in Mexpress database (https://mexpress.be/). The phenotypes analyzed included patient's age, nodal status, tumor stage, ER (estrogen receptor) status; PR (progesterone receptor) status and HER (human epidermal growth factor receptor) status etc. Through cBioportal (http://www.cbioportal.org/) and GSCA (http://bioinfo.life.hust.edu.cn/GSCA/) databases, we further explored the detailed effect of CLMN copy number alteration (CNA) and methylation on its mRNA expression level.

\section{SNV (Single Nucleotide Variation) Analysis of CLMN}

The GSCA database was also used to reveal the single nucleotide variation (SNV) of CLMN in breast invasive carcinoma. In GSCA, we were able to obtain somatic mutation rate of CLMN, mutation class, and SNV class involved in BRCA. In addition, the effect of CLMN SNV on the overall survival of BRCA patients can also be evaluated.

\section{Prognosis Analysis of CLMN}

The Kaplan-Meier Plotter database (http://kmplot.com/) was used to explore the influence of CLMN expression on the prognosis of patients with breast invasive carcinoma. The patients were split by the upper quartile of CLMN expression level, and sample ratio of low/high was $3: 1$. The clinical outcome of patients referred to overall survival (OS), and followup threshold was 10 years (120 months). Restricted survival analysis to subtypes was then performed, and subtypes analyzed included ER status-IHC, PR status-IHC, HER statusarray, Intrinsic subtype, Lymph node status, Grade, and TP53 status. $P<0.05$ was regarded as having statistical significance.

\section{Biological Function Analysis}

Potential function of CLMN involved in breast invasive carcinoma was revealed through functional enrichment analysis containing GO annotation and KEGG pathway analyses. The co-expressed genes with CLMN in breast cancer were first obtained through the LinkedOmics database (http://www.linkedomics.org/). The top 50 co-expressed genes with positive correlation were selected for further enrichment analysis. The WebGestalt database (http:// www.webgestalt.org/) was used to annotate the biological process, molecular function, and cellular component associated with identified co-expressed genes. Significant KEGG pathways of 50 co-expressed genes were detected through KOBAS database (http://kobas.cbi.pku.edu.cn/). Moreover, the potential effect of CLMN on pathway activity of 10 famous cancer-related pathways was explored in GSCA. $P<0.05$ was regarded as having statistical significance. 
A

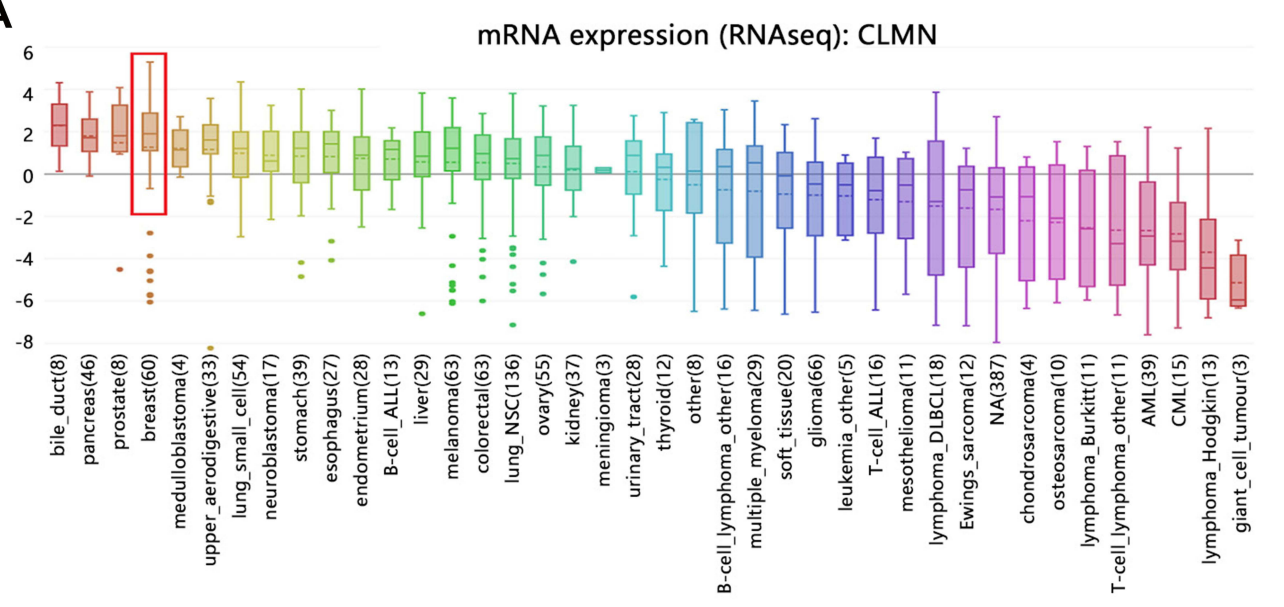

B

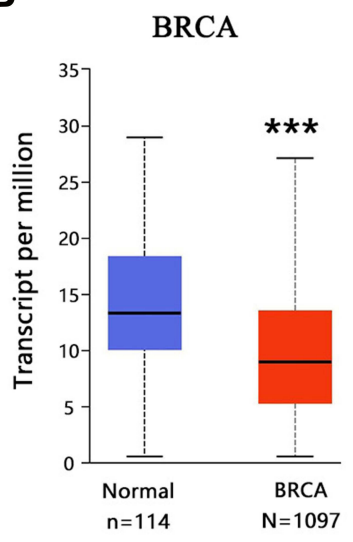

Figure I The mRNA expression of CLMN in BRCA. (A) CLMN expression overview in human cancer cell lines. Red box indicated BRCA. (B) Differential expression of CLMN in normal and breast cancer tissues. $* * * P<0.001$.

\section{Gene Set Level Analysis}

Regarding the top 50 positively correlated significant genes of CLMN, we performed the gene set level analysis in GSCA. Regulation of top 50 genes in BRCA can be found using
GSEA (Gene set enrichment analysis) score. Association between GSVA (Gene Set Variation Analysis) score and activity of cancer-related pathways in BRCA was assessed as well. We also compared GSVA score of inputed gene set between

A

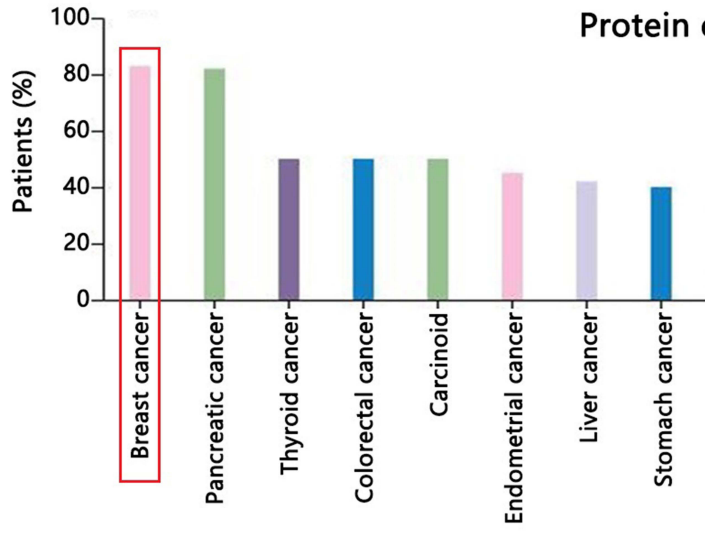

B

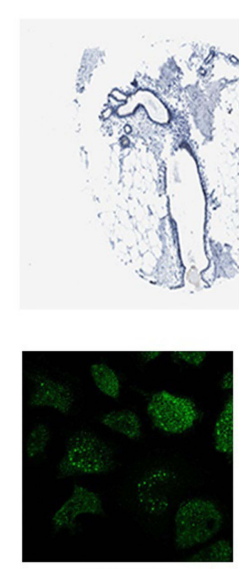

antibody
Normal

HPA012634

Female, age 27

Myoepithelial cells Staining: Medium Intensity: Moderate Quantity: 75\%-25\%

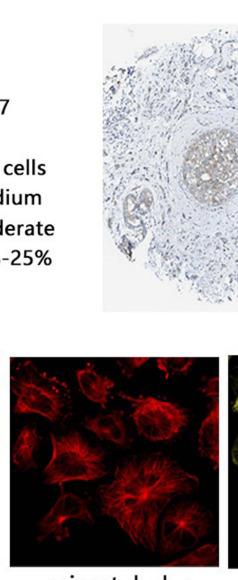

microtubulus

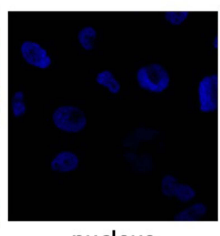

nucleus

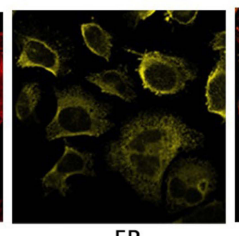

Tumor

HPA012634

Female, age 50

Tumor cells

Staining: Medium

Intensity: Moderate

Quantity: >75\%

Figure 2 Protein expression of CLMN in BRCA. (A) CLMN protein expression overview in human cancers. Red box indicated BRCA. (B) The immunohistochemical images in normal and breast cancer tissues. (C) Protein expression comparison in BRCA and normal tissues. Z-values represented standard deviations from the median across samples. ${ }^{*} P<0.01$. (D) The protein location within cancer cells.

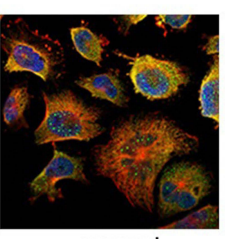

merged

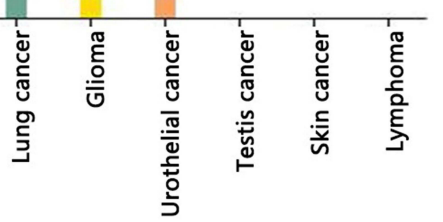

C

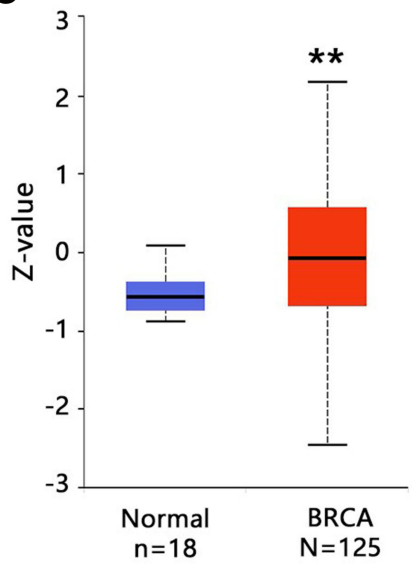


BRCA and normal samples. Relation between GSVA score and TNM stages, cancer subtypes and survival in BRCA was further evaluated.

\section{Results}

\section{The mRNA and Protein Expression of CLMN in BRCA}

The mRNA expression analysis in Figure 1A indicates that relatively higher expression of CLMN was observed in pancreatic, prostate cancer and breast cancer cell lines (red box). The lowest expression refers to giant cell tumor. Quantitative analysis in Figure 1B shows that mRNA expression of CLMN was lower in breast cancer tissue compared with that in normal breast tissue $(P<0.001)$.

Subsequently, protein expression of CLMN in breast cancer was assessed. Figure $2 \mathrm{~A}$ shows that cases with breast cancer exhibited higher protein expression of CLMN. Immunohistochemical images in Figure $2 \mathrm{~B}$ indicate that
CLMN protein staining and intensity were similar between normal and breast cancer cells. But the quantity in tumor cell was higher than in normal cell. Quantitative analysis in Figure $2 \mathrm{C}$ presents a higher expression of CLMN total protein in BRCA than in normal patients $(P<0.01)$. Considering above results, CLMN mRNA expression in BRCA was lower than that in normal patients. However, CLMN protein expression was higher in BRCA than in normal group. Figure 2D shows that CLMN was mainly localized to nuclear bodies, in addition to being localized to the cytosol.

\section{Association Analysis on CLMN mRNA Expression}

From Mexpress analysis, the result indicated that CLMN mRNA expression was statistically associated with patient's age, gender, BRCA progesterone receptor (PR) status, BRCA estrogen receptor (ER) status, histological type, tumor stage, sample type, and copy number (Figure $3 \mathrm{~A}$, all $P<0.05$ ). We
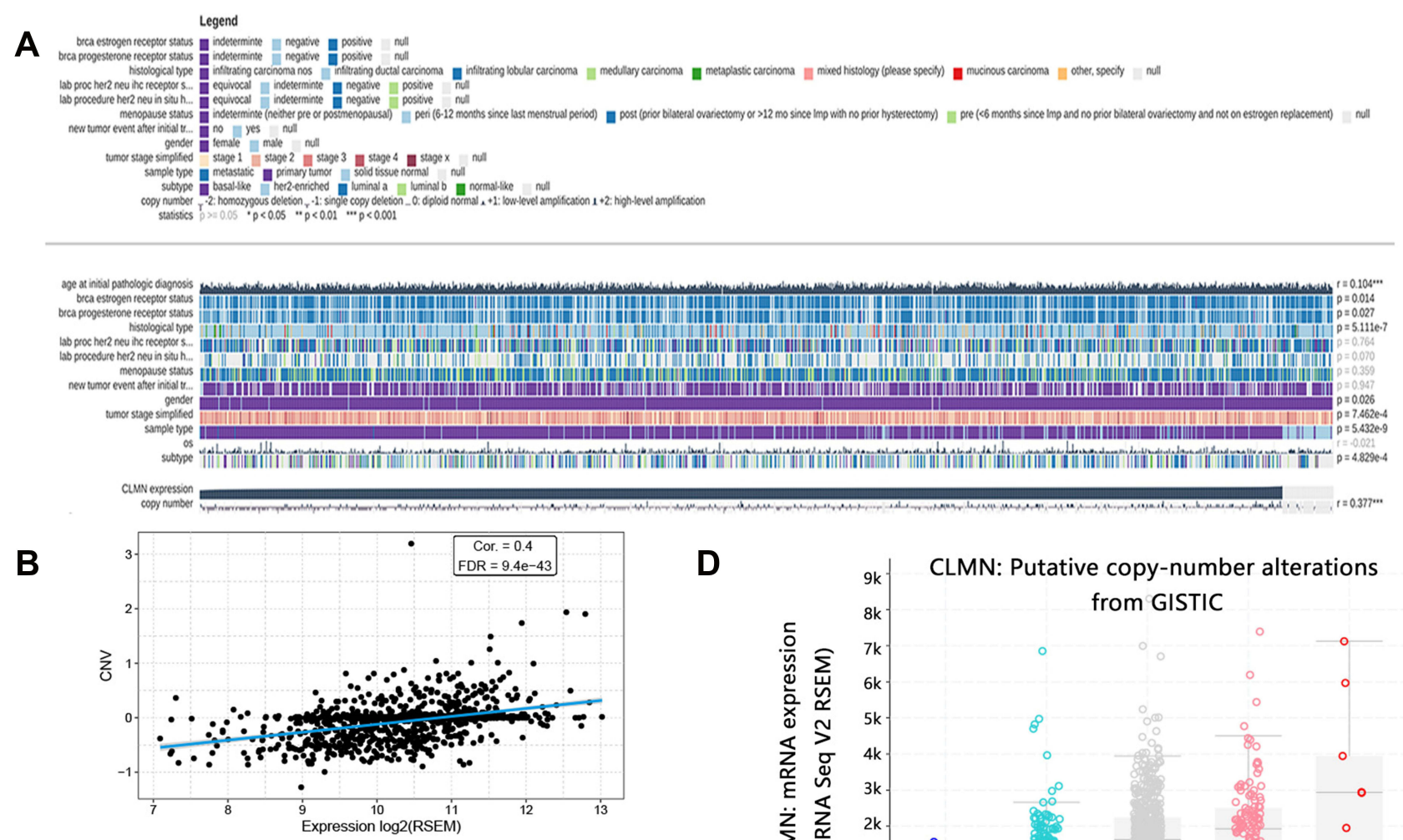

C CNV distribution of CLMN in BRCA tumor samples
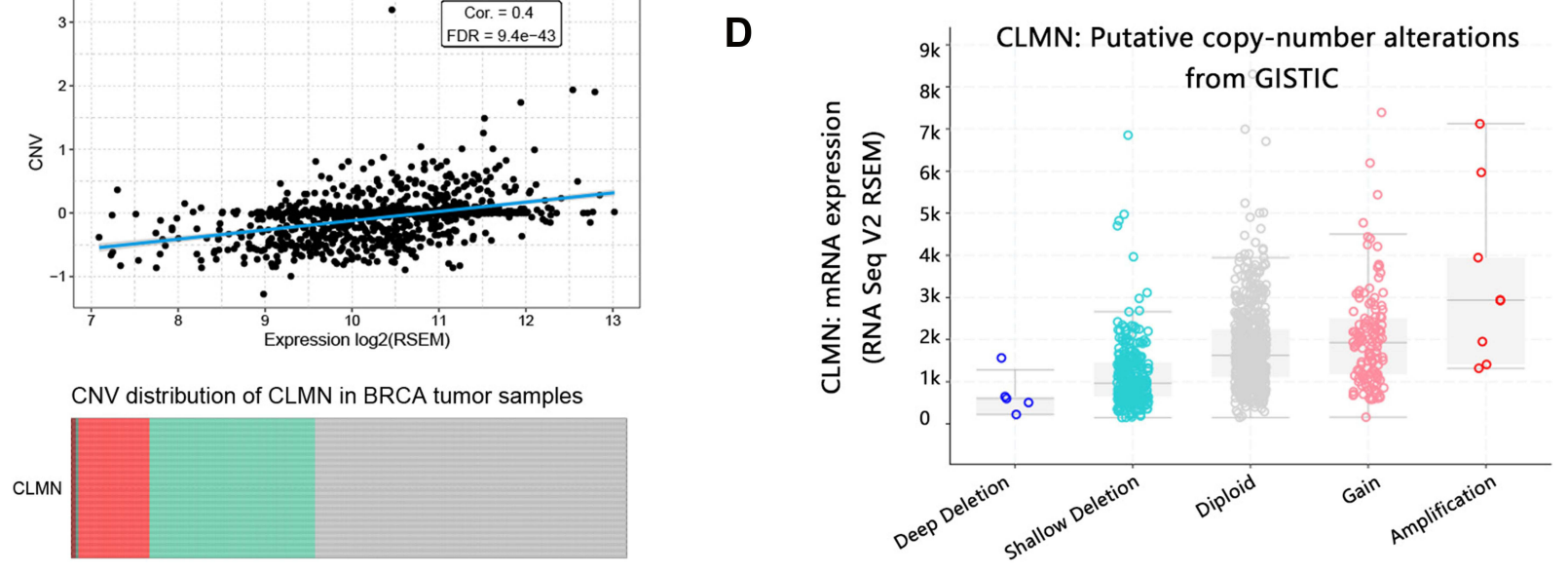

CNV type $\square$ Homo. Amp. $\square$ Homo. Del. $\square$ Hete. Amp. $\square$ Hete. Del. $\square$ None

Figure 3 Related factors affecting CLMN mRNA expression involved in BRCA. (A) Correlation between CLMN expression and clinical data of patients. $* P<0.05$, $* * P<0.01$ and $* * * P<0.001$. (B) Correlation between CLMN expression and CNV. (C) CNV distribution of CLMN. (D) Differential expression of CLMN mRNA among CNV class. Abbreviation: CNV, copy number variation. 
further focused on the effect of copy number variation (CNV) of CLMN on its mRNA expression. A positive relation between CLMN mRNA expression and CNV was observed (Figure 3B). The CNV distribution of CLMN in BRCA is presented at Figure 3C. We further evaluated the relation of CLMN mRNA expression among copy number class, and result showed that deep deletion type caused a lower CLMN mRNA expression (Figure 3D).

We subsequently explored the correlation between CLMN mRNA expression and methylation. Result in Figure 4A shows that CLMN mRNA expression was negatively correlated to its methylation level in BRCA (all $P<0.001$ ). Differential expression analysis showed that CLMN methylation level in BRCA was lower than that in normal group (Figure 4B, $P<0.0001$ ). However, the methylation level of CLMN exerted no effect on the overall survival of BRCA patients (Figure 4C).

\section{The SNV (Single Nucleotide Variation) Analysis of CLMN}

Due to the copy number alteration of CLMN, we subsequently explored the single nucleotide variation of CLMN

A

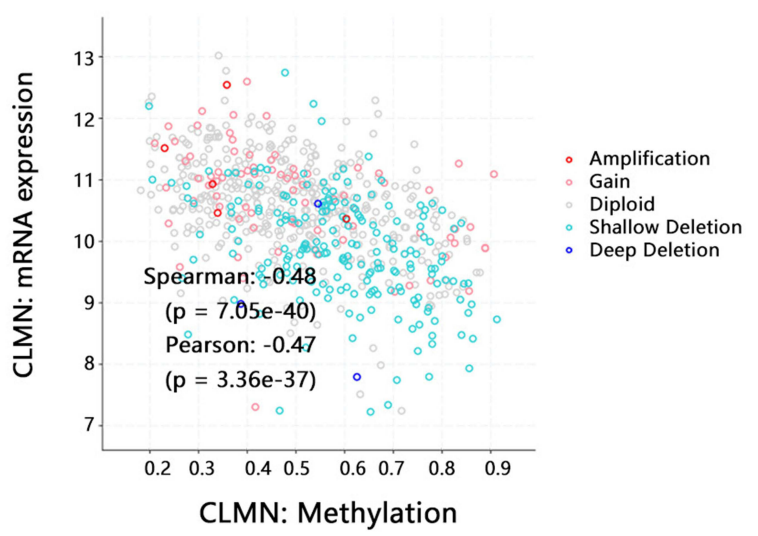

B

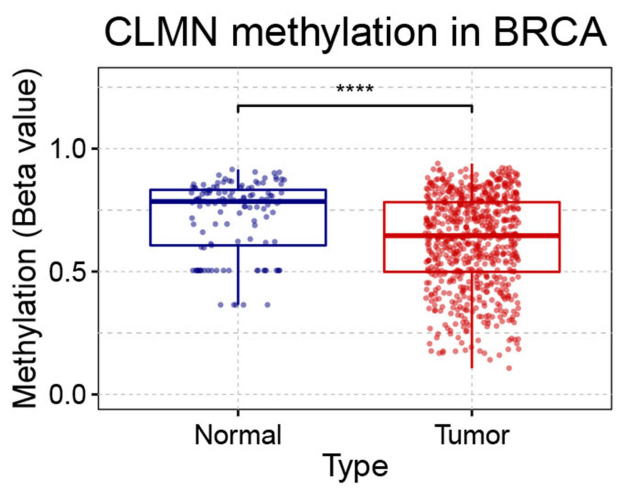

in breast invasive carcinoma. As observed from Figure 5A, the somatic mutation rate of CLMN was $0.88 \%$. Variant classification was mainly presented as missense mutation (Figure 5B), and the main class for $\mathrm{SNV}$ was $\mathrm{C}>\mathrm{T}$ (Figure 5C). Survival analysis showed that higher risk of death referred to CLMN mutant group but showed no statistical significance (Figure 5D).

\section{The Prognosis Analysis of CLMN}

The Figure 6A presents the influence of CLMN expression on the 10-years overall survival of patients with breast cancer. Survival analysis indicated that high expression of CLMN prolonged the survival time of patients and led to a favorable prognosis $(\mathrm{HR}=0.67, P=0.0011)$. A restrict survival analysis on CLMN expression was further performed and presented in Table 1 and Figure 6C. For patients with ER positive status, the difference in survival time between CLMN high and low expression groups was statistically significant $(\mathrm{HR}=0.53, P=0.0042)$. Survival differences between the two groups were also observed in patients with negative HER status ( $\mathrm{HR}=0.75, P=0.0043)$. The expression of CLMN was statistically associated with
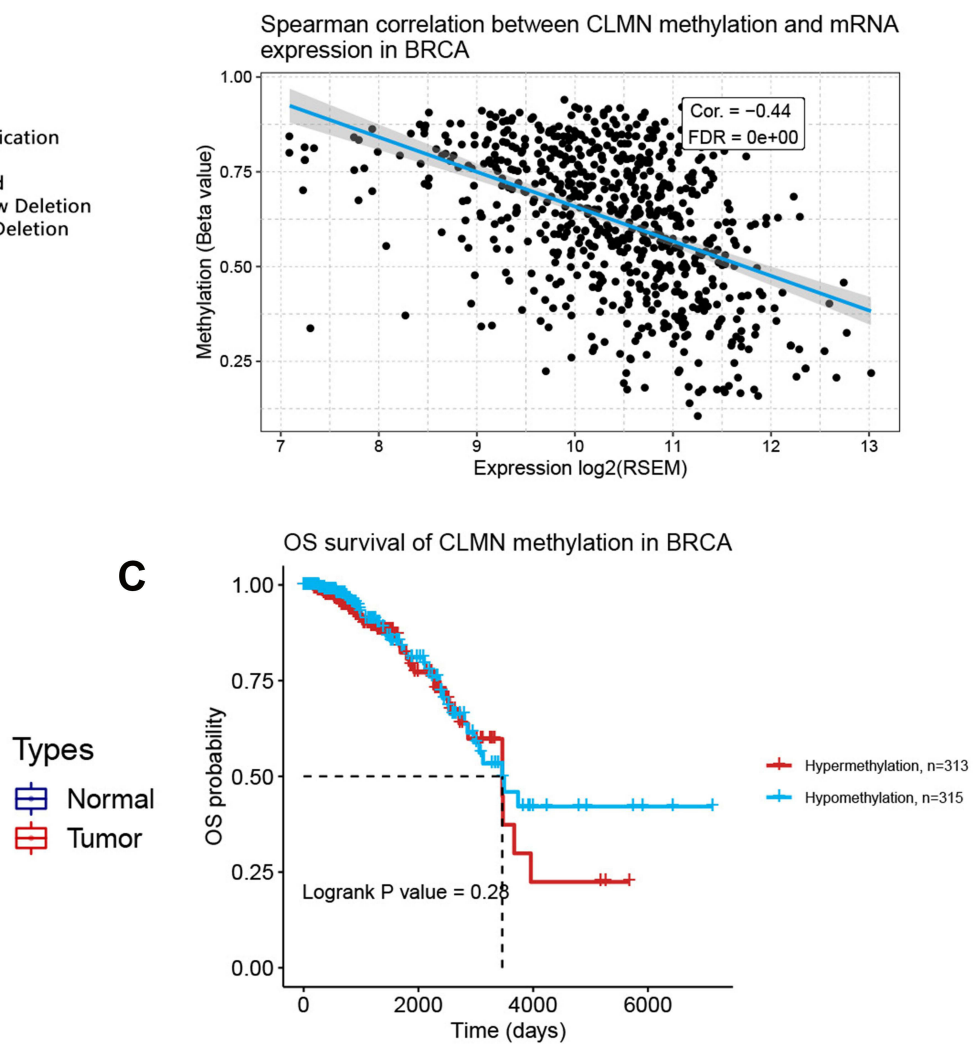

Figure 4 CLMN methylation analysis in BRCA. (A) Correlation between CLMN mRNA expression and methylation level. (B) Differential expression of CLMN methylation in normal and BRCA patients. $* * * * P<0.0001$. (C) Correlation between $C L M N$ methylation level and overall survival of patients. 
A

CLMN: Somatic Mutation Rate: $\mathbf{0 . 8 8 \%}$

$\left.\begin{array}{l}5 \\ \\ \\ \end{array}\right]$

- Missense mutation

- Nonsense mutation

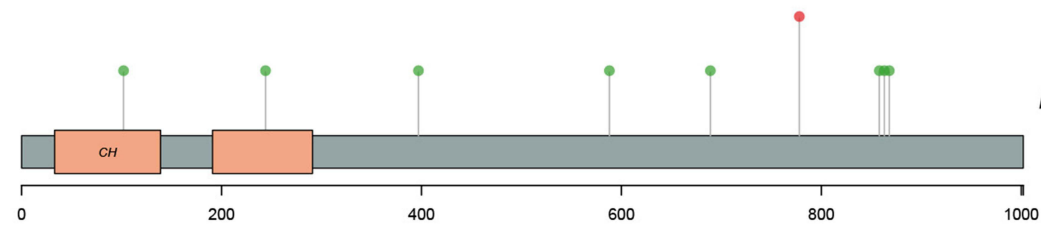

C SNV Class

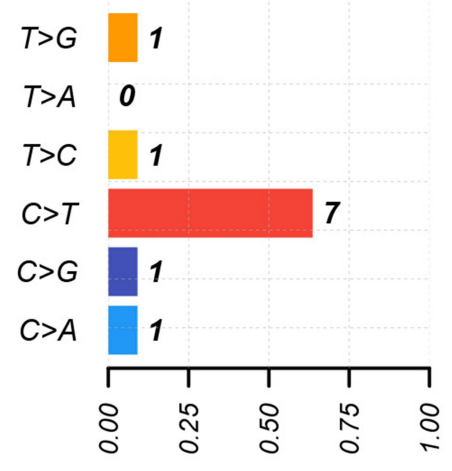

B

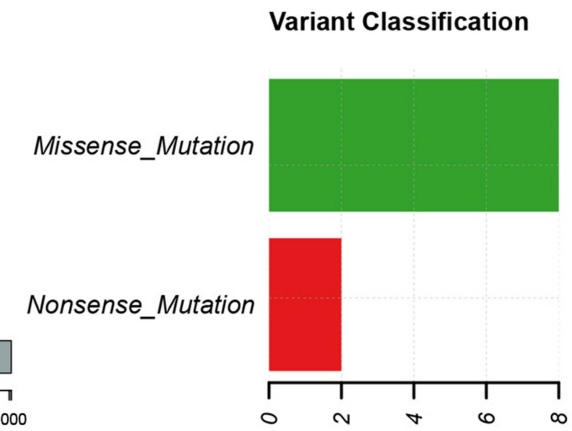

D

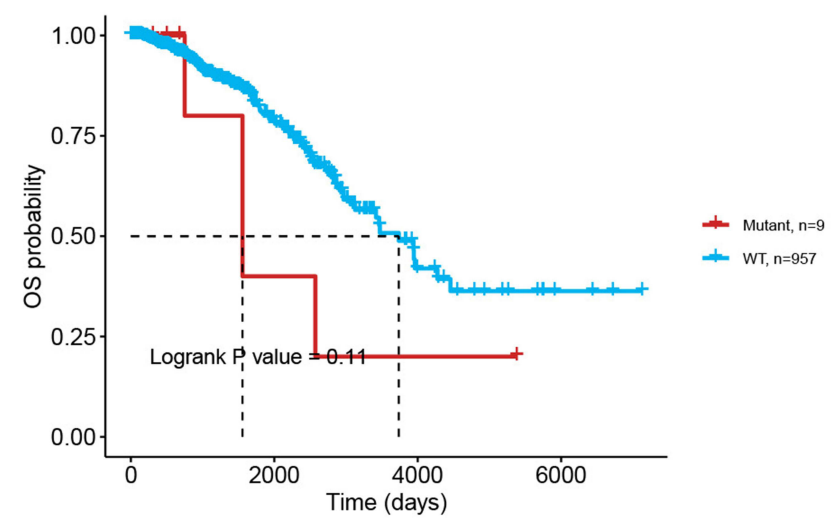

Figure 5 SNV summary and its correlation with survival in BRCA. (A) Somatic mutation of CLMN in BRCA. (B) Variant classification. (C) SNV class. (D) Impact of CLMN SNV on overall survival in BRCA.

Abbreviation: SNV, single nucleotide variation.

the prognosis of patients with luminal $\mathrm{A}(\mathrm{HR}=0.54$, $P=0.0065)$ and luminal $\mathrm{B}(\mathrm{HR}=0.54, P=0.0123)$ subtypes. Positive lymph node status resulted in the survival difference between high and low expression groups $(\mathrm{HR}=0.56$, $P=0.0069)$. The PR status, tumor grade, and TP53 status did not affect the survival difference of patients (all $P>0.05)$. We also focused on the survival of triple negative breast cancer patients, and result indicated that CLMN expression did not correlate with these patient's overall survival (Figure 6B).

\section{Functional Enrichment Analysis}

In order to determine the potential biological function of CLMN, the co-expressed genes with CLMN in breast invasive carcinoma were first obtained. Figure 7A shows the related genes associated with CLMN in breast cancer with volcano plot. The top 50 positively and 50 negatively correlated genes with CLMN are presented in Figure 7B with heat map. The Figure $7 \mathrm{C}$ analyzes the relation between CLMN and co-expressed genes with highest correlation coefficient, and TRIP11 showed strong positive correlation with CLMN. The identified 50 co-expressed genes with positive correlation were selected for further functional enrichment analysis.

The biological function of 50 co-expressed genes with positive correlation was annotated from aspects of biological process, cellular component, and molecular function. Figure 8 shows that genes were mainly located at nucleus and membrane. The participated biological process included metabolic process and biological regulation. The molecular function was involved in protein binding and ion binding.

Significant KEGG pathway analysis on identified 50 co-expressed genes was detected through KOBAS database. The enriched terms are visualized in Figure 9A with bubble plot. Each bubble represented an enriched function, and the size of the bubble was set with six gradients according to the $\mathrm{p}$-value, from small to large, representing 
A

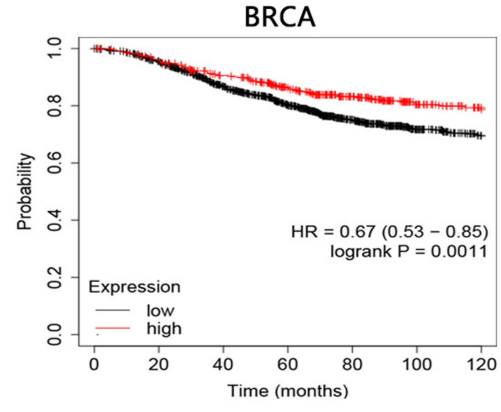

B

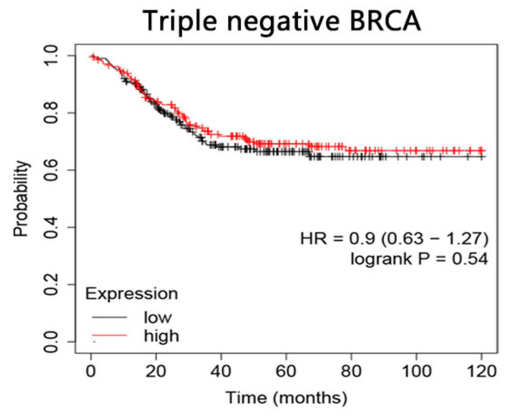

C

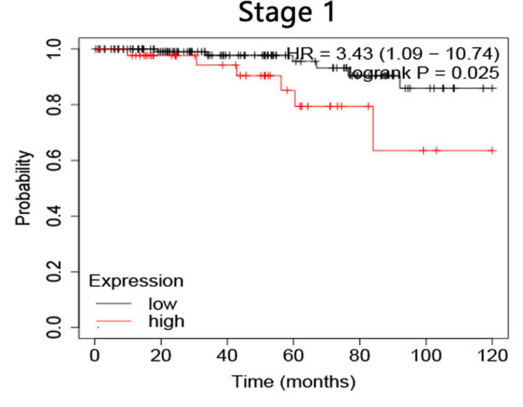

Luminal A

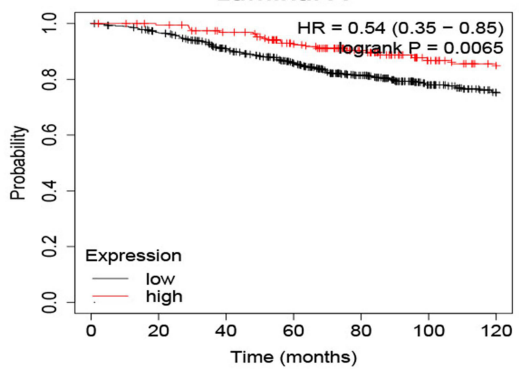

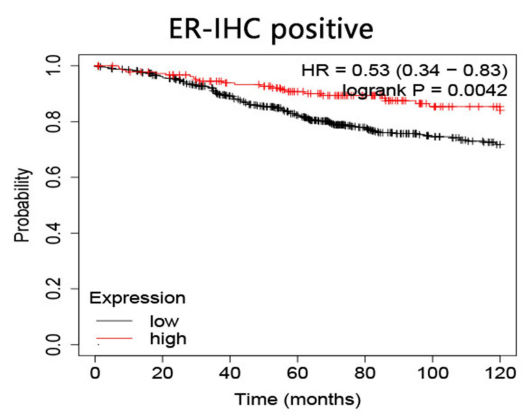

Luminal B

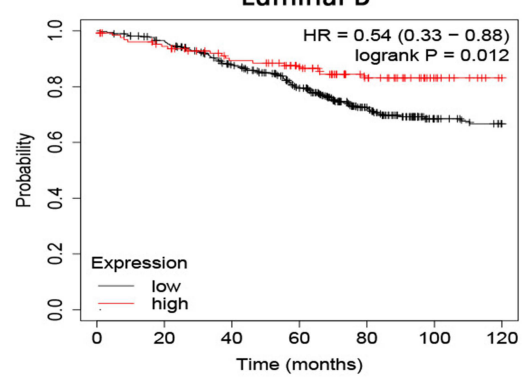

HER2 negative
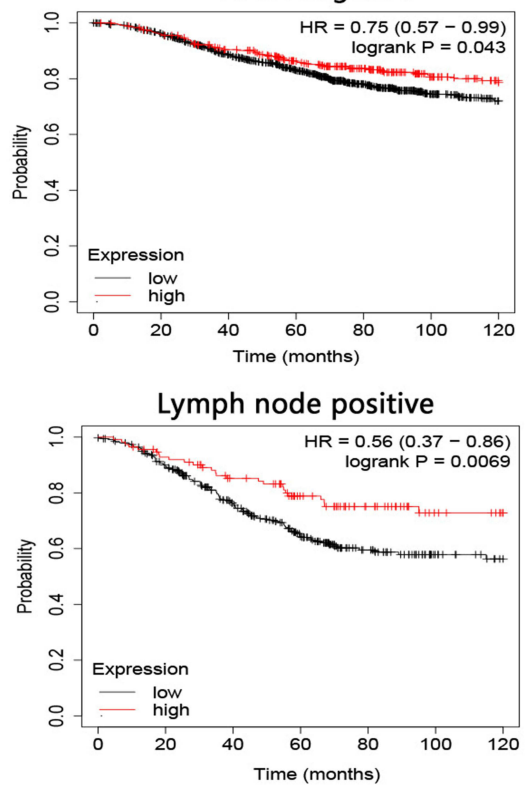

Figure 6 The 10-year overall survival analysis on CLMN in BRCA. (A) Survival analysis on BRCA patients without restricted criteria. (B) Survival analysis on triple negative breast cancer patients. (C) Restrict overall survival analysis based on subtypes of BRCA.

Abbreviations: ER, estrogen receptor; HER2, human epidermal growth factor receptor-2; HR, hazard ratio.

different significance levels. Pathway analysis indicated that co-expressed genes mainly participated in cell cycle, oocytes meiosis, metabolic pathways, progesteronemediated oocyte maturation, and pyrimidine metabolism. The correlation between CLMN mRNA expression and pathway activity (Figure 9B) showed that CLMN exerted inhibition effect on cell cycle and apoptosis pathways. However, pathway activity score of 2 pathways showed no statistical significance between CLMN high and low expression group (Figure 9C).

\section{Gene Set Level Analysis}

We then performed gene set analysis on the top 50 positively correlated significant genes of CLMN. The result in Figure 10A shows that among the input gene set, half of them were downregulated in BRCA, and distributed on the right blue side. GSVA analysis in Figure 10B presents detailed information on the difference in pathway activity between high and low GSVA score groups in BRCA. The results showed that pathway activities of cell cycle negatively correlated with GSVA score of gene set. While hormone ER pathway activity was significantly and positively related to the GSVA score. The Spearman correlation between GSVA score and pathway activity of cell cycle (Figure 10C) as well as hormone ER (Figure 10D) was further analyzed.

We further explored the detailed GSVA score of gene set containing 50 genes in BRCA. Differential expression analysis presented that GSVA score of gene set in BRCA was lower than in normal patients (Figure 11A, $P<0.001$ ). The GSVA score was significantly associated with BRCA subtype (Figure 11B, $P<0.001$ ) and cancer stage (Figure $11 \mathrm{C}, P<0.001$ ). Survival analysis showed that higher risk was observed in lower GSVA group, but showing no statistical significance (Figure 11D, $P<0.001$ ). 
Table I 10-Years Survival Difference Analysis Between CLMN Expression Groups (High vs Low)

\begin{tabular}{|c|c|c|c|c|}
\hline & Subtypes & N (Low/High) & HR (95\% Cl) & $P$-value \\
\hline \multirow[t]{3}{*}{ Tumor grade } & Grade I & $13 \mid / 44$ & $0.55(0.16-1.89)$ & 0.3348 \\
\hline & Grade 2 & $333 / 110$ & $0.63(0.37-1.08)$ & 0.0879 \\
\hline & Grade 3 & $440 / 146$ & $1.31(0.94-1.84)$ & 0.1114 \\
\hline \multirow[t]{4}{*}{ Cancer stage } & I & $135 / 45$ & $3.43(1.09-10.74)$ & 0.0250 \\
\hline & 2 & $464 / 155$ & $0.75(0.40-\mid .4 I)$ & 0.3700 \\
\hline & 3 & $|86 / 6|$ & $0.76(0.35-1.63)$ & 0.4800 \\
\hline & 4 & $15 / 5$ & $0.29(0.06-1.35)$ & 0.0960 \\
\hline \multirow[t]{2}{*}{ Gender } & Female & $808 / 269$ & $0.85(0.57-1.28)$ & 0.4400 \\
\hline & Male & I & Too low sample & I \\
\hline \multirow[t]{2}{*}{ ER status-IHC } & Positive & $566 / 188$ & $0.53(0.34-0.83)$ & 0.0042 \\
\hline & Negative & $390 / 130$ & $1.04(0.7|-| .52)$ & 0.8400 \\
\hline \multirow[t]{2}{*}{ PR status-IHC } & Positive & $117 / 39$ & $0.58(0.22-1.53)$ & 0.2636 \\
\hline & Negative & $219 / 73$ & $0.98(0.56-1.70)$ & 0.9411 \\
\hline \multirow[t]{2}{*}{ HER status-array } & Positive & $315 / 105$ & $0.78(0.50-1.22)$ & 0.2787 \\
\hline & Negative & $1095 / 364$ & $0.75(0.57-0.99)$ & 0.0433 \\
\hline \multirow[t]{4}{*}{ Intrinsic subtype } & Basal & $303 / 101$ & $1.39(0.93-2.09)$ & 0.1096 \\
\hline & Luminal $\mathrm{A}$ & $596 / 198$ & $0.54(0.35-0.85)$ & 0.0065 \\
\hline & Luminal B & $386 / 129$ & $0.54(0.33-0.88)$ & 0.0123 \\
\hline & HER2+ & $124 / 42$ & $1.26(0.68-2.3 \mathrm{I})$ & 0.4626 \\
\hline \multirow[t]{2}{*}{ Lymph node status } & Positive & $339 / 113$ & $0.56(0.37-0.86)$ & 0.0069 \\
\hline & Negative & $544 / 182$ & $0.78(0.5 \mathrm{I}-1.20)$ & 0.2600 \\
\hline \multirow[t]{2}{*}{ TP53 status } & Mutated & $63 / 21$ & $1.27(0.50-3.20)$ & 0.6140 \\
\hline & Wild type & $23 / 8$ & $1.59(0.42-5.94)$ & 0.4897 \\
\hline
\end{tabular}

Note: Bold value showed the terms with $P$-value $<0.05$.

Abbreviations: ER, estrogen receptor; PR, progesterone receptor; HER2, human epidermal growth factor receptor-2; N, sample number; HR, hazard ratio; Cl, confidence interval.

\section{Discussion}

Calmin (CLMN) belongs to all-trans retinoic acid (atRA)regulated gene, and few studies investigated its significance in human cancers up to now. The present study initially found that CLMN was down-regulated in patients with BRCA, and its expression was associated with the patient's age, nodal metastases status, cancer stages, and TP53 mutation status. The genetic alteration of CLMN was observed in BRCA patients and the protein change appeared in R244Q, as well as missense substitution showed the highest frequency of mutation. Among BRCA patients, CLMN high expression was conducive to their overall survival in terms of positive ER statusIHC, negative HER status-array, luminal A and luminal B subtypes, as well as positive lymph node status. The infiltration level of immune cells also influenced the survival time of patients.
So far, there have been few studies reporting the function of CLMN in cancers. Previous study identified CLMN as a vitamin D target gene in human breast cancer tissue, and elevated expression of CLMN was associated with prolonged relapse-free survival for breast cancer patients. $^{7}$ Another study also confirmed CLMN as 1 alpha, 25-dihydroxyvitamin D responsive in human breast tumor, suggesting that CLMN may prove useful as a biomarker of vitamin $\mathrm{D}$ exposure in breast tissue. ${ }^{8}$ CLMN has been determined as a potential candidate tumor suppressor gene of nasopharyngeal carcinoma. ${ }^{9}$ The present study found that patients with CLMN high expression showed a favorable prognosis, suggesting its significant value as a tumor suppressor gene. The influence of CLMN expression on the progression of breast cancer needs to be confirmed through molecular biology experiments. It has been found that cancer stage $(P=0.004)$, ER 

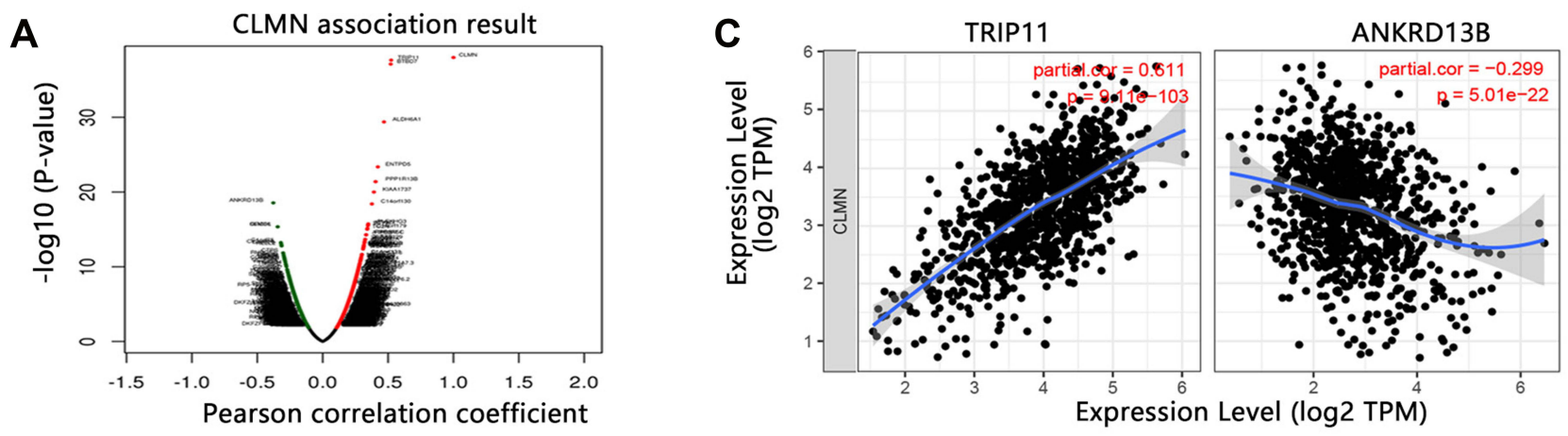

B
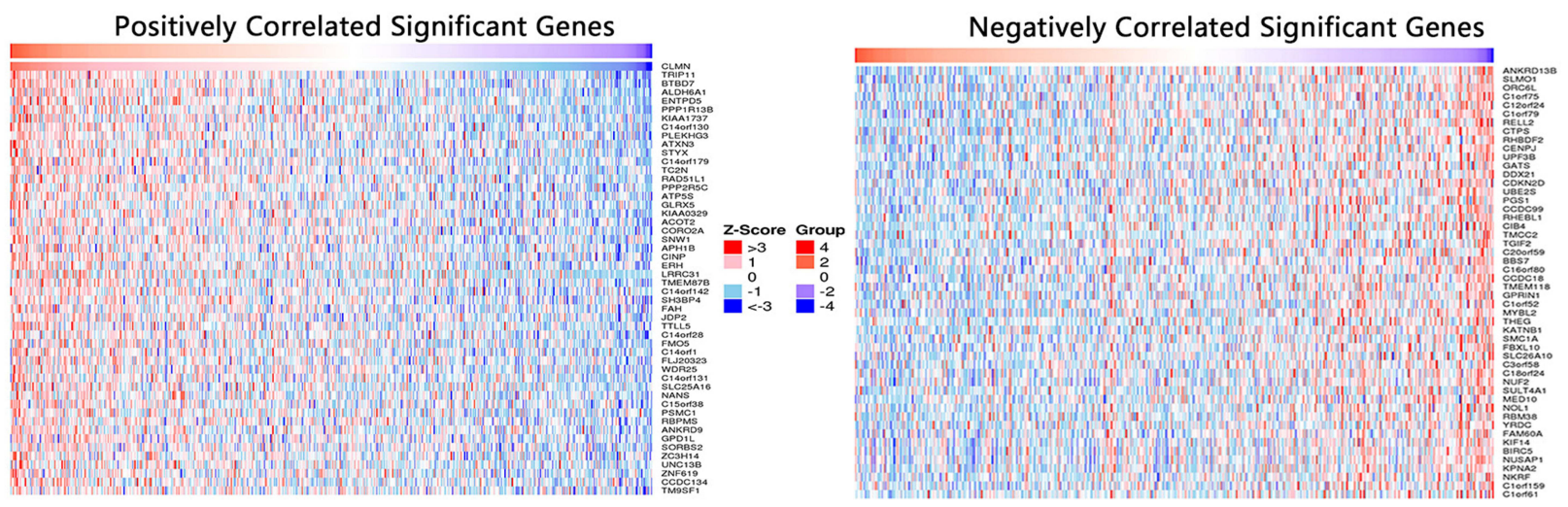

Figure 7 The co-expressed genes with CLMN in BRCA. (A) Volcano plot. (B) Heat map showing the top 50 genes with positive or negative correlation. (C) Association between CLMN and genes with highest positive or negative correlation coefficient.
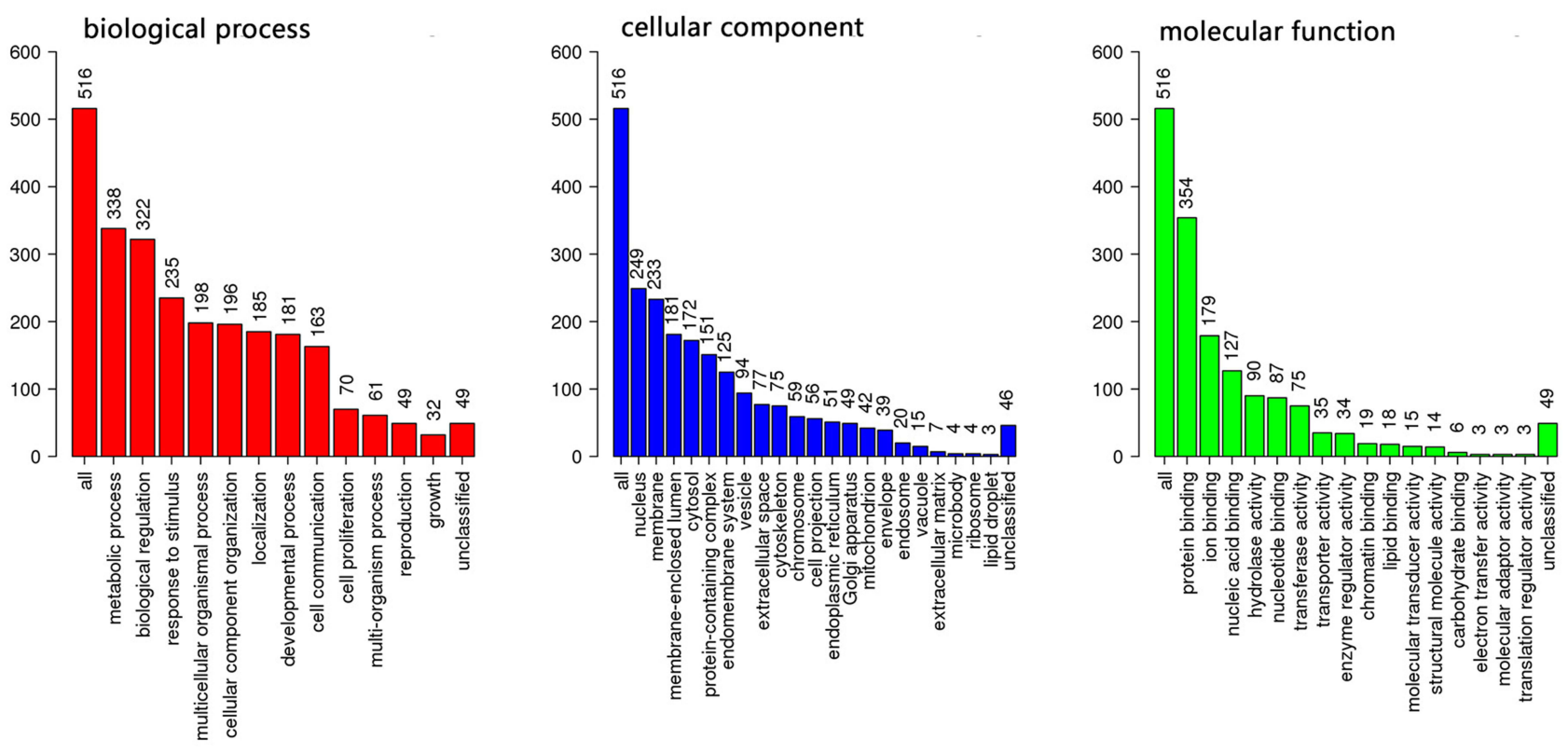

Figure 8 The GO annotation analysis associated with 50 positively co-expressed genes. 
A

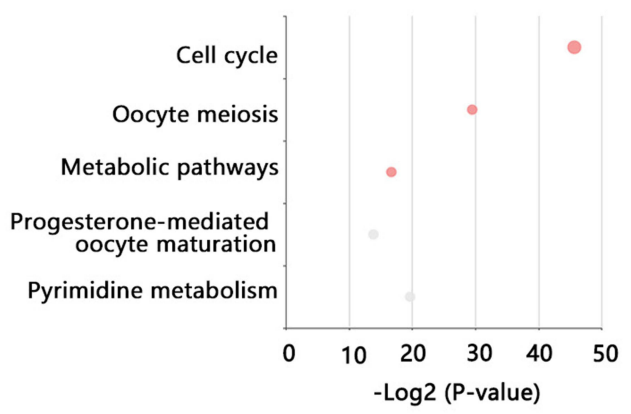

C

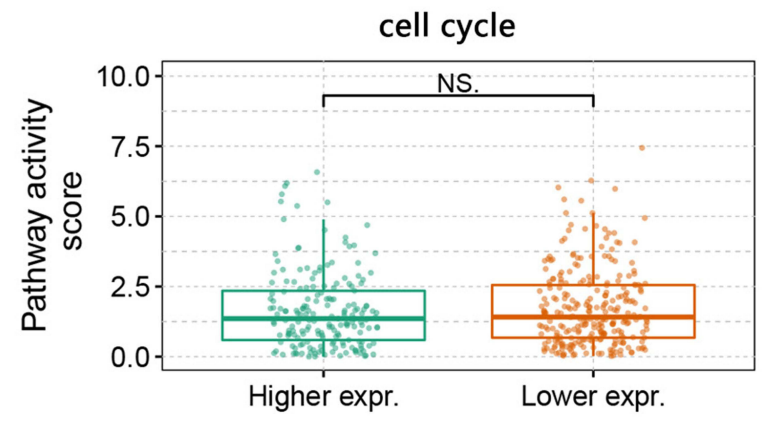

B
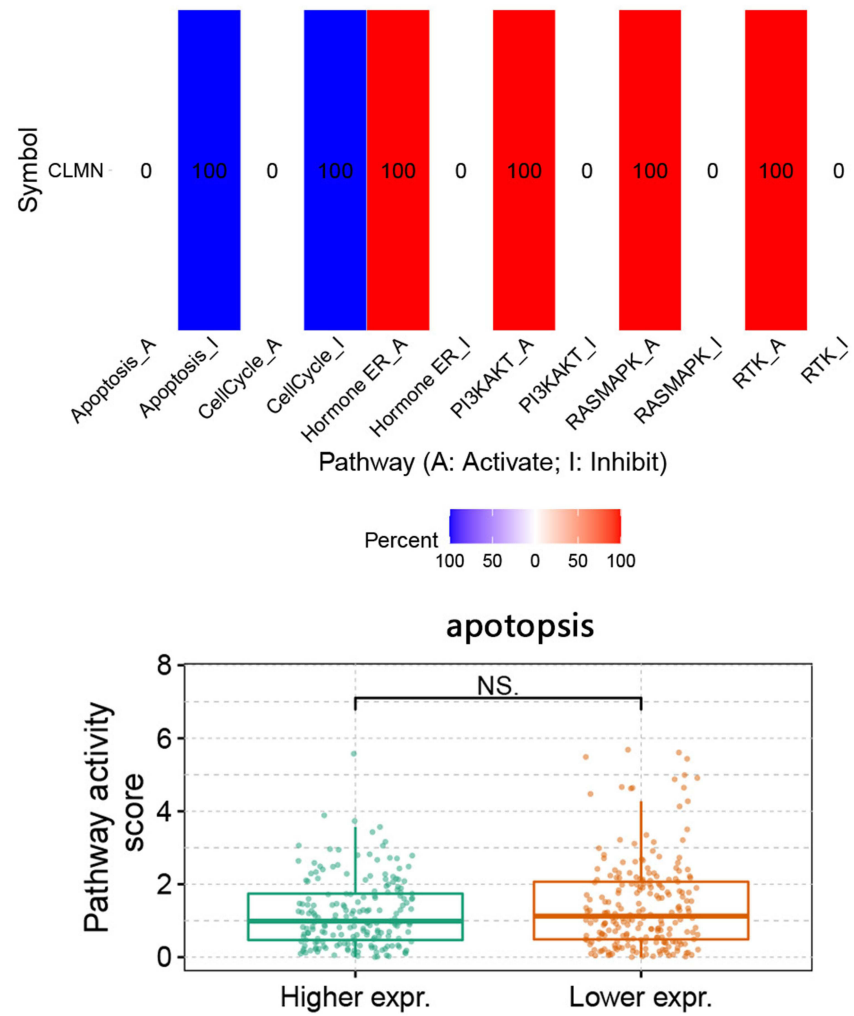

Figure 9 The biological pathway analysis on the top 50 co-expressed genes of CLMN with positive correlation. (A) Top 5 KEGG significant pathways. (B) Potential effect of CLMN on pathway activity in BRCA. The number in each cell indicated the percentage of cancers. Because we just focused on BRCA, therefore the number was presented as 100. (C) Activity of cell cycle and apoptosis pathways between high and low CLMN expression groups in BRCA.

Abbreviation: NS, no significance.

( $P=0.028), \mathrm{PR}(P=0.018)$ status, and subtype $(P=0.025)$ were significantly associated with breast cancer recurrence. ${ }^{10}$ Triple-negative breast cancer, higher stage, and higher histological grade were associated with earlier brain metastases. ${ }^{11}$ The survival analysis in this study indicated that the clinical outcome between CLMN high and low expression groups was statistically associated with ER, HER, and lymph node status, as well as subtypes.

Pathway analysis indicated that CLMN possibly participated in cell cycle, oocytes meiosis, and metabolic pathways. Previous study indicated that increase of CLMN mRNA induced growth inhibition and neuronal differentiation of neuroblastoma cells, as well as CLMN promoted exit from the cell cycle, a requisite event for neuronal differentiation. ${ }^{12}$ TRIP11 was identified as significant gene with a strong correlation with CLMN. TRIP11 was a multifunctional protein localizing either to Golgi apparatus or in the nucleus, acting as coactivator of transcription mediated by thyroid hormone receptor (THR) and hypoxia-induced factor (HIF). It was observed that TRIP11 expressions correlated with tumor grades of renal cell cancer and decreased expression correlated with poor survival of patients, which might be associated with triiodothyronine signaling pathway. ${ }^{13}$ The present study confirmed the poor prognosis of patients with CLMN low expression, suggesting the possibility of triiodothyronine signaling pathway involvement in breast cancer. The metabolic etiology of breast cancer has been explored during the past years using metabolomics. Several important metabolic pathways, such as taurine and hypotaurine metabolism and the alanine, aspartate, and glutamate pathway, have been revealed as critical biological pathways for early diagnosis of breast cancer. ${ }^{14}$ A study combining transcriptomic and metabolomics data found that dysregulation of purine metabolism pathway affected the progression of breast cancer. ${ }^{15}$ Targeting the ceramide metabolic pathway could induce apoptosis of breast cancer cell lines. ${ }^{16}$ It followed that metabolic pathways played a vital role in the progression of cancers. The regulation of CLMN associated with metabolic pathways in breast cancer required further investigations. 
A

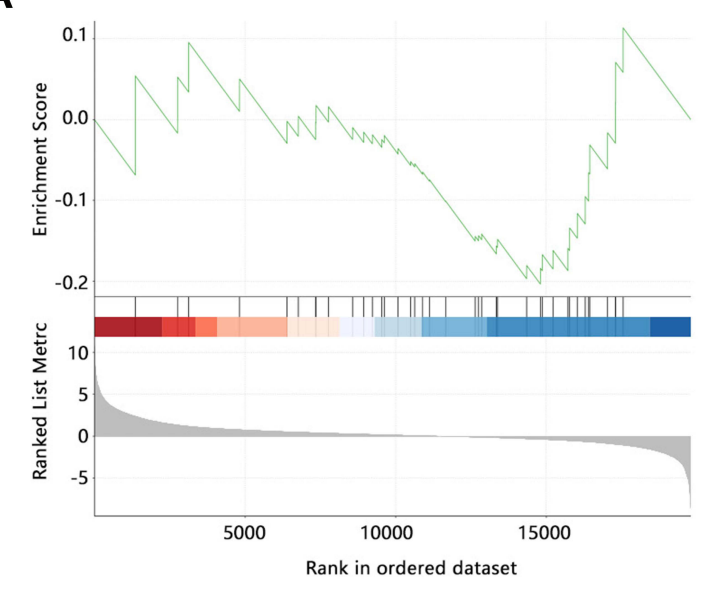

C

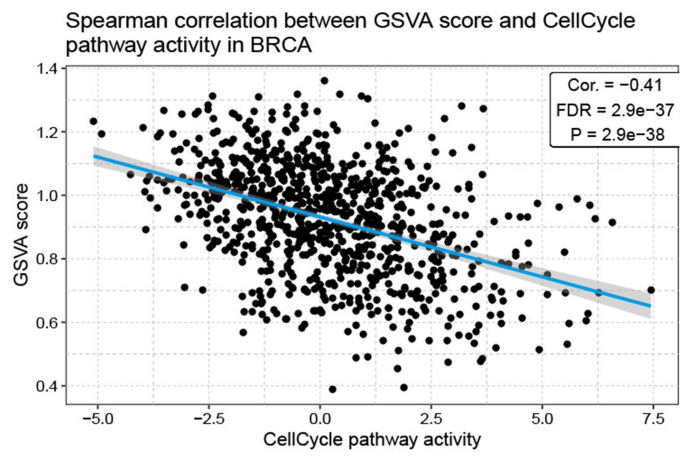

B

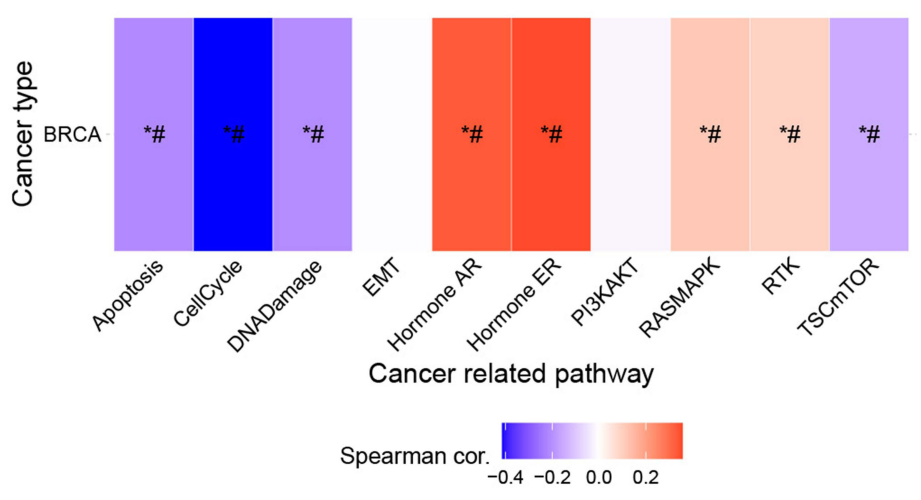

D

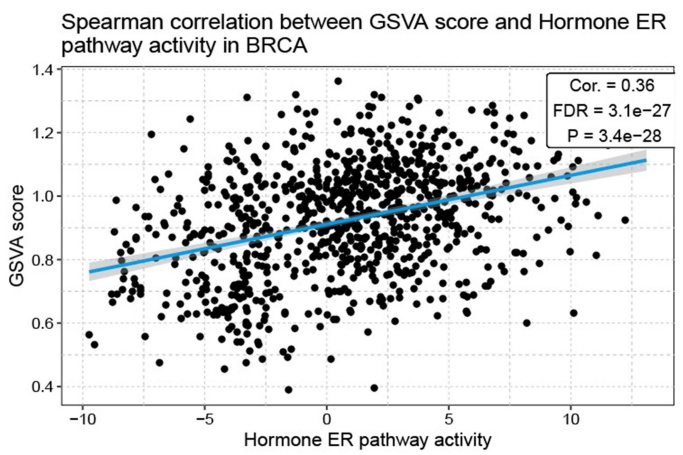

Figure 10 Gene set level analysis on 50 positively correlated significant genes of CLMN in BRCA. (A) GSEA score of gene set in BRCA. (B) Association between GSVA score and activity of cancer related pathways in BRCA. ${ }^{* P}<0.05,{ }^{\#} \mathrm{FDR}<0.05$. And Spearman correlation between GSVA score and (C) Cell cycle, (D) Hormone ER pathway activities in BRCA.

Abbreviations: GSEA, gene set enrichment analysis; GSVA, gene set variation analysis; ER, estrogen receptor; FDR, false discovery rate.

Our study presently shows the clinical significance of CLMN in BRCA, but several limitations should be mentioned. The CLMN mRNA and protein expressions lack the experimental verification in vitro. It is better if the prognostic impact of CLMN can be confirmed in clinical cohort. Conducting more experiments on this subject will enlighten the unclear aspects of cancer diagnosis and treatment. Although this study presented several limitations, its potential clinical value cannot be ignored. This work can help researchers in the field to further investigate the role of CLMN in different aspects of breast and possibly other types of cancers.

\section{Conclusions}

The present study initially explored the potential function of CLMN in breast invasive carcinoma. Expression of
CLMN mRNA was lower in BRCA than in normal patients. We also found that mRNA expression of CLMN was associated with BRCA patient's age, gender, PR status, ER status, histological type, tumor stage, sample type, copy number, and methylation level. Survival analysis showed that CLMN low expression caused an unfavorable prognosis, but high expression exerted a positive effect on the survival of patients. Further analysis indicated that survival difference between CLMN high and low expression groups can be observed in patients with stage 1 , positive ER, negative HER, luminal A, luminal B, or positive lymph node status. Enrichment analysis showed that CLMN mainly participated in cell cycle and exerted inhibition effect on this pathway involved in BRCA, but CLMN expression value did not influence the pathway activity of cell cycle. 
A

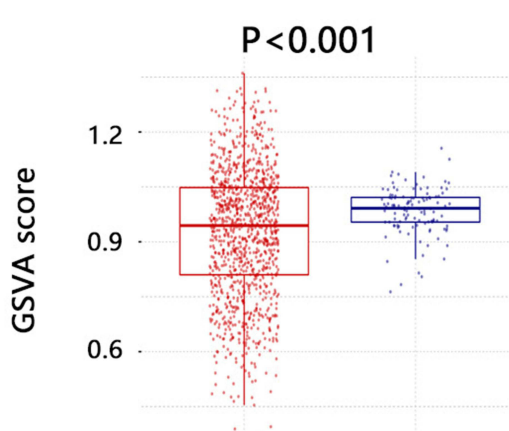

BRCA Normal

\section{C}

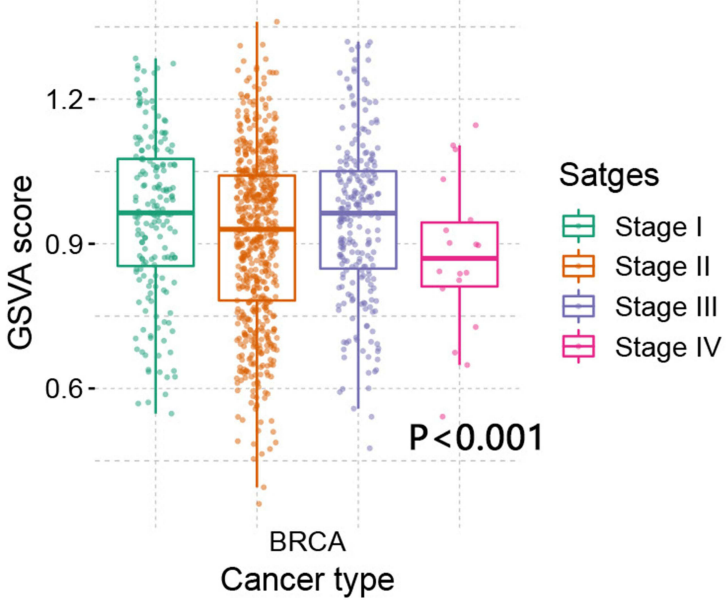

B

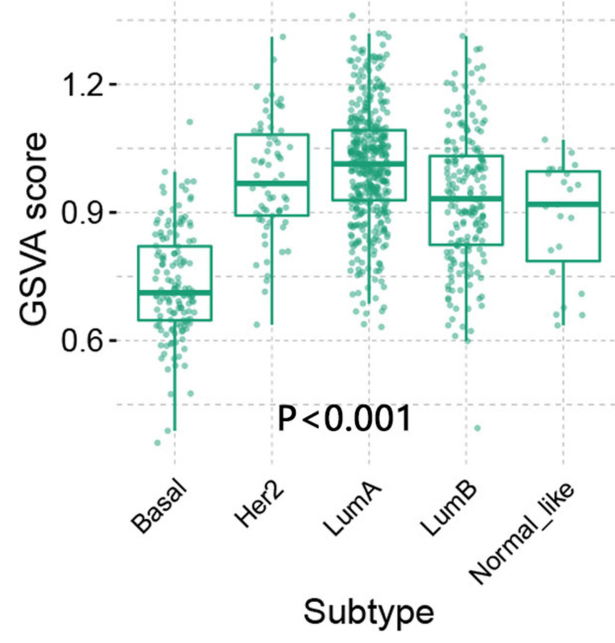

D

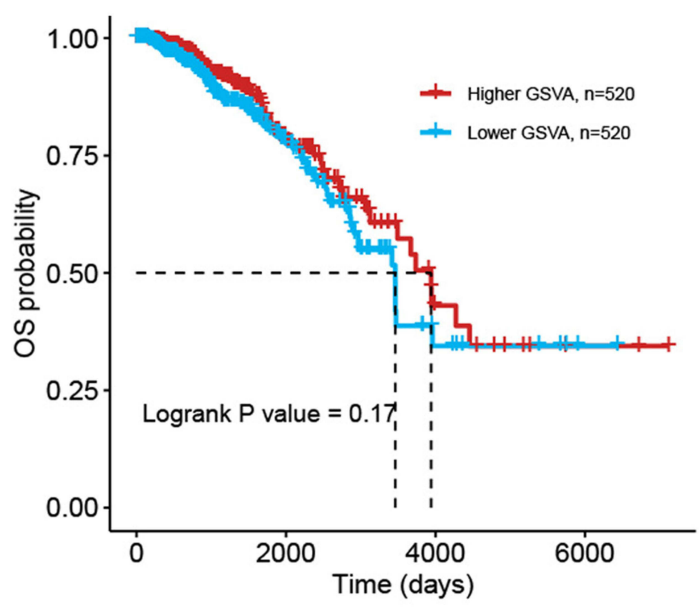

Figure II Comparison analysis of GSVA score in BRCA. (A) Differential GSVA in normal and BRCA patients. (B) GSVA score between subtypes. (C) GSVA score among stages. (D) Overall survival difference between GSVA score groups.

Abbreviations: GSVA, gene set variation analysis.

\section{Data Sharing Statement}

The data used and/or analyzed during the current study are available from the corresponding author on reasonable request.

\section{Funding}

There is no funding to report.

\section{Disclosure}

The authors declare that they have no competing interests.

\section{References}

1. Siegel RL, Miller KD, Jemal A. Cancer statistics, 2020. CA Cancer $J$ Clin. 2020;70:7-30. doi:10.3322/caac.21590

2. Hurvitz SA, Hu Y, O’Brien N, Finn RS. Current approaches and future directions in the treatment of HER2-positive breast cancer. Cancer Treat Rev. 2013;39:219-229. doi:10.1016/j.ctrv.2012.04.008
3. Sun X, Wang Z, Chen X, Shen K. CRISPR-cas9 screening identified lethal genes enriched in cell cycle pathway and of prognosis significance in breast cancer. Front Cell Dev Biol. 2021;9:646774. doi:10.3389/fcell.2021.646774

4. Merrill RA, Ahrens JM, Kaiser ME, Federhart KS, Poon VY, ClagettDame M. All-trans retinoic acid-responsive genes identified in the human SH-SY5Y neuroblastoma cell line and their regulated expression in the nervous system of early embryos. Biol Chem. 2004;385:605-614. doi:10.1515/BC.2004.075

5. Marzinke MA, Henderson EM, Yang KS, See AW, Knutson DC, Clagett-Dame M. Calmin expression in embryos and the adult brain, and its regulation by all-trans retinoic acid. Dev Dyn. 2010;239:610-619. doi:10.1002/dvdy.22171

6. Takaishi M, Ishisaki Z, Yoshida T, Takata Y, Huh NH. Expression of calmin, a novel developmentally regulated brain protein with calponin-homology domains. Brain Res Mol Brain Res. 2003;112:146-152. doi:10.1016/S0169-328X(03)00061-5

7. Sheng L, Anderson PH, Turner AG, et al. Identification of vitamin D3 target genes in human breast cancer tissue. J Steroid Biochem Mol Biol. 2016;164:90-97. doi:10.1016/j.jsbmb.2015.10.012

8. Simmons KM, Beaudin SG, Narvaez CJ, Welsh J. Gene signatures of 1,25-Dihydroxyvitamin D3 exposure in normal and transformed mammary cells. J Cell Biochem. 2015;116:1693-1711. doi:10.1002/jcb.25129 
9. Yanatatsaneejit $\mathrm{P}$, Chalermchai $\mathrm{T}$, Kerekhanjanarong V, et al. Promoter hypermethylation of CCNA1, RARRES1, and HRASLS3 in nasopharyngeal carcinoma. Oral Oncol. 2008;44:400-406. doi:10.1016/j.oraloncology.2007.05.008

10. Badal K, Ali R, Warner WA, et al. Factors associated with breast cancer recurrence and survival at Sangre Grande Hospital, Trinidad. Cancer Causes Control. 2021;32(7):763-772. doi:10.1007/s10552021-01427-z

11. Cao TQ, Dixit K, Santa-Maria C, Kumthekar P. Factors affecting time to brain metastases for stage 2 and 3 breast cancer patients: a large single-institutional analysis with potential screening implications. Neurooncol Adv. 2021;3:vdab009.

12. Marzinke MA, Clagett-Dame M. The all-trans retinoic acid (atRA)-regulated gene Calmin (Clmn) regulates cell cycle exit and neurite outgrowth in murine neuroblastoma (Neuro2a) cells. Exp Cell Res. 2012;318:85-93. doi:10.1016/j.yexcr.2011.10.002
13. Poplawski P, Piekielko-Witkowska A, Nauman A. The significance of TRIP11 and T3 signalling pathway in renal cancer progression and survival of patients. Endokrynol Pol. 2017;68:631-641.

14. Huang S, Chong N, Lewis NE, Jia W, Xie G, Garmire LX. Novel personalized pathway-based metabolomics models reveal key metabolic pathways for breast cancer diagnosis. Genome Med. 2016;8:34. doi:10.1186/s13073-016-0289-9

15. Luo $\mathrm{X}, \mathrm{Yu} \mathrm{H}$, Song $\mathrm{Y}$, Sun T. Integration of metabolomic and transcriptomic data reveals metabolic pathway alteration in breast cancer and impact of related signature on survival. J Cell Physiol. 2019;234:13021-13031. doi:10.1002/jcp.27973

16. Vethakanraj HS, Babu TA, Sudarsanan GB, Duraisamy PK, Ashok Kumar S. Targeting ceramide metabolic pathway induces apoptosis in human breast cancer cell lines. Biochem Biophys Res Commun. 2015;464:833-839. doi:10.1016/j.bbrc.2015.07.047
International Journal of General Medicine

\section{Publish your work in this journal}

The International Journal of General Medicine is an international, peer-reviewed open-access journal that focuses on general and internal medicine, pathogenesis, epidemiology, diagnosis, monitoring and treatment protocols. The journal is characterized by the rapid reporting of reviews, original research and clinical studies
Dovepress

across all disease areas. The manuscript management system is completely online and includes a very quick and fair peer-review system, which is all easy to use. Visit http://www.dovepress.com/ testimonials.php to read real quotes from published authors. 